Artículo

\title{
Estabilidad de rendimiento y características fisicoquímicas de grano de híbridos de maíz en Valles Altos de México
}

\author{
María Gricelda Vázquez-Carrillo ${ }^{1}$ \\ Aarón Martínez-Gutiérrez ${ }^{2}$ \\ Benjamín Zamudio-González ${ }^{1 \S}$ \\ Alejandro Espinosa-Calderón ${ }^{1}$ \\ Margarita Tadeo-Robledo ${ }^{3}$ \\ Antonio Turrent Fernández ${ }^{1}$
}

${ }^{1}$ Campo Experimental Valle de México-INIFAP. Carretera Los Reyes-Texcoco km 13.5, Coatlinchan, Texcoco, Estado de México. CP. 56250. (gricelda_vazquez@yahoo.com; espinoale@yahoo.com.mx; aturrent37@yahoo.com.mx) ${ }^{2}$ División de Estudios de Posgrado e Investigación-Instituto Tecnológico del Valle de Oaxaca (ITVO). (aaron_0715@hotmail.com). ${ }^{3}$ Facultad de Estudios Superiores CuautitlánUniversidad Nacional Autónoma de México. (tadeorobledo@yahoo.com.mx).

${ }^{\S}$ Autor para correspondencia: bzamudiog @ yahoo.com.mx.

\section{Resumen}

Las cosechas de grano de maíz en Valles Altos de México en lo general no cumplen con los requisitos de calidad para su uso en la industria de la masa y la tortilla. Nuevos híbridos han sido liberados por centros de investigación para estas condiciones agroecológicas y es necesario evaluar la estabilidad de producción y calidad de grano en diferentes ambientes de producción para evitar rechazos en el mercado. El objetivo de este estudio fue identificar híbridos estables en su rendimiento, características fisicoquímicas de los granos y calidad de tortillas. Se evaluaron diez híbridos en cinco ambientes diferentes con cuatro repeticiones para cada tratamiento. Los datos se analizaron con SAS, versión 9.0, se desarrolló el Anova y la comparación de medias se hizo con la prueba de Tukey al 5\%. Se detectaron diferencias significativas para híbridos, ambiente y la interacción híbrido*ambiente; para rendimiento de grano, variables físico-químicas de las tortillas. Los rendimientos de grano oscilaron entre 9 a $12.4 \mathrm{t} \mathrm{ha}^{-1}$. La mayor estabilidad por adaptabilidad y rendimiento por ambientes se encontró para los híbridos H-70, H-66 y Tsiri Puma. Dentro del grupo de los granos duros se encuentran el Tsiri Puma y el testigo comercial Albatros. Los híbridos Atziri Puma, en Temascalcingo y H-50 en Atlacomulco, presentaron los granos más grandes. Se encontraron correlaciones entre proteínas $v s$ aceite $(\mathrm{r}=0.6)$, aceite $v s$ almidón $(\mathrm{r}=-0.86)$ y proteínas vs almidón ( $\mathrm{r}=-0.82)$. El mayor rendimiento en masa fue el híbrido H-51 AE, seguido de H-47 AE, Albatros y Tsiri Puma con $2 \mathrm{~kg}$ de masa por kg de maíz nixtamalizado. El rendimiento y calidad de tortillas de los híbridos evaluados cumplen con los valores demandados por la industria. Los resultados de esta investigación permiten recomendar estos nuevos híbridos, por su estabilidad en el rendimiento, en los ambientes de prueba, calidad comercial del grano y calidad de sus tortillas.

Palabras clave: almidón, híbrido-ambiente, híbridos e índice de flotación.

Recibido: junio de 2020

Aceptado: octubre de 2020 


\section{Introducción}

En México, el maíz (Zea mays L.) es el cultivo más importante en la dieta de los mexicanos, principalmente como tortillas. La región de los Valles Altos de México (> $2200 \mathrm{~m}$ ) se siembran en condiciones de 'punta de riego', humedad residual o temporal, más de dos millones de hectáreas de maíz, las cuales representan cerca de $25 \%$ de la superficie nacional (SIAP, 2018). Los maíces sembrados corresponden principalmente a maíces nativos $(80 \%)$ con rendimiento promedio bajo $\left(<3.14 \mathrm{t} \mathrm{ha}^{-1}\right)$ y variedades mejoradas cuyos granos no cumplen con los requisitos mínimos para su uso en la industria de la masa y la tortilla (Vázquez et al., 2016), tornándose grises, lo que limita la aceptación del producto por el consumidor (Salinas et al., 2012).

Se requieren estudios de la calidad del grano de nuevos híbridos de maíces liberados en Valles Altos, que satisfagan los requerimientos de la industria de la masa y la tortilla (Gaytán et al., 2013) y que contribuyan a que los productores de esta región posicionen su producción de grano de maíz en las industrias de masa-tortilla (IMT) y la de harina nixtamalizada (IHN). El rendimiento y las propiedades físicas y químicas del grano de maíz son influenciados por el factor híbrido (G), ambiente (A) y la interacción G×A. Vázquez et al. (2016); Lozano et al. (2015) evaluaron diferentes híbridos para conocer su adaptabilidad y estabilidad en el rendimiento, las características fisicoquímicas del grano y la calidad de la tortilla en condiciones ambientales contrastantes. Según Vázquez et al. (2016) para los industriales de la masa y la tortilla es importante que los híbridos mantengan su estabilidad en el rendimiento y calidad del grano en los diferentes ambientes de producción evitando ajustes en el procesamiento.

Entre varios procedimientos para estimar el efecto de híbrido, ambiente, y la interacción híbrido ambiente (IGA), ha probado ser exitosa la metodología del análisis de regresión en los sitios (SREG) (Crossa et al., 2015), la cual permite estimar la estabilidad, evaluar localidades y clasificar ambientes por medio de una gráfica bidimensional (biplot) de híbridos y ambientes (Lozano et al., 2015). La identificación de variedades mejoradas que presenten estabilidad de rendimientos y parámetros de calidad de grano y sus tortillas, contribuye a una mayor aceptación por parte de los agricultores y la industria. El objetivo de este estudio fue identificar híbridos estables en su rendimiento, características fisicoquímicas de sus granos y calidad de tortillas entre 10 híbridos de maíz evaluados en cinco ambientes de Valles Altos del Estado de México.

\section{Materiales y métodos}

Este trabajo se realizó en el ciclo agrícola primavera-verano 2016 en cinco localidades del Estado de México, a saber, por sitio y fecha de siembra: Temascalcingo (TEM) el 3 de mayo; Atlacomulco (ATL) el 5 de abril; Jocotitlán (JOC) el 13 de abril; Ixtlahuaca (IXT) el 23 de abril; y en Jilotepec (JIL), el 25 de mayo. Las características ambientales y fisicoquímicas del suelo de las localidades se encuentran en Martínez et al. (2018).

Se evaluaron siete híbridos formados por el Instituto Nacional de Investigaciones Forestales, Agrícolas y Pecuaria (INIFAP): H-66, H-70, H-50, H-47AE, H-49AE, H-51AE y H-53AE; dos híbridos, por la Facultad de Estudios Superiores de Cuautitlán (FESC-UNAM): Atziri-Puma (ATZ) y Tsiri-Puma (TP), ambos de versión androestéril; y un testigo: Albatros (ALB), de uso comercial por productores de Valles Altos. 
La fertilización al suelo fue de 250-60-60 de NPK + mezcla de micronutrientes en dos etapas. A la siembra se fertilizó con 100-60-40 de NPK y el resto de nitrógeno $(150 \mathrm{~N})$ se aplicó entre etapa $\mathrm{V}_{4-10}$, fraccionado en partes iguales en función a la humedad del suelo. El diseño de los tratamientos se formó al combinar el factorial de cinco ambientes $\times 10$ híbridos, con cuatro repeticiones, considerando como fuentes de variación los híbridos, ambientes y sus interacciones .

\section{Rendimiento y características físicas-químicas del grano}

El rendimiento del peso seco de grano $(\mathrm{RG})$ fue calculado ( $\mathrm{t} \mathrm{ha}^{-1}$ ), ajustado a $14 \%$ de humedad y extrapolado por una hectárea. En laboratorio se midió índice de flotación (IF, medida indirecta de dureza) (SE, 2002), peso de 100 granos (PCG) (medida indirecta de tamaño) y peso hectolítrico (PH) (Salinas y Vázquez, 2006). Se cuantificó aceite (ACE), proteína (PRO) y almidón (ALM) de acuerdo con los métodos 30-25.01, 46-16.01 y 76-13.01 del AACCI (2000).

\section{Nixtamalización y elaboración de tortillas}

El proceso de nixtamalización consistió en la cocción de una muestra de 500 g de maíz, con $3.5 \mathrm{~g}$ de $\mathrm{Ca}(\mathrm{OH})_{2}$ y $1 \mathrm{~L}$ de agua. El tiempo de cocción se asignó acorde a su dureza: $\mathrm{IF}=0$-12 (Muy duro), 45 min; 13-37 (Duro), 40 min; 38-62 (Medio), 35 min; 63-87 (Suave), 30 min; 87-100 (Muy suave), 25 min (SE, 2002). El nixtamal reposó en la solución de cocción (nejayote) 18 h, lavándose posteriormente con agua $(0.5 \mathrm{~L})$. En el nejayote se cuantificó pérdida de materia seca (PMS). En un molino de piedras Fumasa ${ }^{\circledR}$ Mod. MN-80 Universal (Fumasa SA., Mex.) se molió el nixtamal con agua (5\% volumen/peso), para obtener la masa (Vázquez et al., 2012). La masa se acondicionó hasta que una esfera de $10 \mathrm{~g} \pm 0.02$ comprimida por $5 \mathrm{~s}$ con una báscula de capacidad de $2.5 \mathrm{~kg}$ alcanzara un diámetro de $5 \mathrm{~cm}$, o sea, $58 \% \pm 0.05$ de humedad. La masa se troqueló para obtener tortillas de $12 \mathrm{~cm}$ de diámetro y $1.25 \mathrm{~mm}$ de espesor, mismas que se cocieron por $1.5 \mathrm{~min}$ en una tortilladora Villamex ${ }^{\circledR}$ Mod. V-14 C/R (Villamex, Mex.) con tres comales (temperatura promedio $=265^{\circ} \mathrm{C}$ ).

Las tortillas una vez frías $\left(25^{\circ} \mathrm{C}\right)$ se empacaron en bolsas de polietileno con cierre hermético. Con los pesos registrados de nixtamal, masa acondicionada y tortillas frías se calcularon los rendimientos. En las tortillas recién elaboradas (30 min) (RE), de 24 (HT24) y 48 (HT48) horas de almacenamiento a $4{ }^{\circ} \mathrm{C}$ se midió: humedad (AACC, 2000), fuerza de ruptura (FZA) y elongación máxima (EL) con un texturometro Brookfield ${ }^{\circledR}$ CT3 (Middleboro, EU) (Vázquez et al., 2015) a velocidad de avance de $1 \mathrm{~mm} \mathrm{~s}^{-1}$. Con FZA y EL se calculó el módulo de elasticidad de Young (Y) (Mao et al., 2002) con la ecuación $\mathrm{Y}=\mathrm{E} / \mathrm{D}$, donde el esfuerzo $\mathrm{E}=\mathrm{FZA}$ *área transversal del disco de tortilla; $\mathrm{D}=$ diámetro de la tortilla usado para la evaluación*EL.

\section{Análisis estadístico}

Se realizó un análisis de varianza (Anova), donde $\mathrm{HIB}=$ efecto fijo, $\mathrm{AMB}=$ aleatorio y la interacción HIB $\times$ AMB; pruebas $(p<0.05)$ de comparación de medias (Tukey) y análisis de correlación (Pearson). Las variables con interacción significativa (HIB $\times$ AMB), se obtuvo el biplot SREG en el que se graficaron los efectos principales de híbrido (CP1) y los de la interacción (CP2) (Vázquez et al., 2016; Crossa et al., 2015; Lozano et al., 2015) con el uso del paquete estadístico Statistical Analysis System (SAS) versión 9.0 (SAS Inst., 2002). 


\section{Resultados y discusión}

El análisis de varianza combinado detectó diferencias significativas para el factor híbrido, ambiente y la interacción híbrido-ambiente (IGA) $(p \leq 0.05)$, en rendimiento de grano, variables físicas $(\mathrm{PH}$, PCG e IF), características químicas (ACEI, PROT, ALM) (Cuadro 1) y de tortillas (HT, HT24, HT48, FZAHT, FZA24, FZA48, YRE, Y24, Y48). El rendimiento promedio de los híbridos fue mayor a la media anual (riego + temporal) estatal $\left(4.3 \mathrm{t} \mathrm{ha}^{-1}\right)$ y nacional (3.9 $\left.\mathrm{t} \mathrm{ha}^{-1}\right)$ (SIAP, 2018). El PH superó al demandado en la norma NMX-032/1 (SE, 2002), con densidad mínima de 74 kg $\mathrm{hL}^{-1}$, con endospermo duro e intermedio (IF entre 13-37 y 38-62 respectivamente). Los contenidos de ACE, PRO y ALM, se encuentran dentro del rango reportado para maíces dentados (Watson, 2003). El híbrido Atziri Puma fue el de mayor RG, PCG y PRO, pero el de menor contenido de ALM (Cuadro 1).

Cuadro 1. Valores promedio de rendimiento, características físicas y químicas de grano de 10 híbridos de maíz evaluados en cinco ambientes de Valles Altos del Estado de México. Primaveraverano 2016.

\begin{tabular}{cccccccc}
\hline Híbridos & RG $\left(\mathrm{t} \mathrm{ha}^{-1}\right)$ & PH $\left(\mathrm{kg} \mathrm{hL}^{-1}\right)$ & PCG $(\mathrm{g})$ & $\mathrm{IF}$ & Aceite $^{\S}$ & Proteína $^{\S}(\%)$ & Almidón $^{\S}$ \\
\hline H-51AE & $10.4 \mathrm{~d}$ & $77.6 \mathrm{e}$ & $36.1 \mathrm{~h}$ & $62 \mathrm{a}$ & $5.1 \mathrm{~cd}$ & $10.1 \mathrm{bc}$ & $69.6 \mathrm{bcd}$ \\
H-47AE & $11.2 \mathrm{c}$ & $77.3 \mathrm{f}$ & $36.1 \mathrm{~h}$ & $60 \mathrm{a}$ & $4.9 \mathrm{e}$ & $9.9 \mathrm{~d}$ & $70 \mathrm{a}$ \\
H-66 & $11.6 \mathrm{abc}$ & $77.2 \mathrm{f}$ & $37.9 \mathrm{f}$ & $54 \mathrm{~b}$ & $5.4 \mathrm{a}$ & $9.8 \mathrm{~d}$ & $69.5 \mathrm{~cd}$ \\
Atziri Puma & $12 \mathrm{a}$ & $77.2 \mathrm{f}$ & $39.9 \mathrm{a}$ & $51 \mathrm{bc}$ & $5.3 \mathrm{ab}$ & $10.3 \mathrm{a}$ & $69.1 \mathrm{e}$ \\
H-50 & $11.5 \mathrm{abc}$ & $77.5 \mathrm{e}$ & $39.2 \mathrm{~b}$ & $49 \mathrm{bcd}$ & $5.1 \mathrm{~cd}$ & $10.2 \mathrm{ab}$ & $69.4 \mathrm{de}$ \\
H-70 & $11.6 \mathrm{abc}$ & $78.4 \mathrm{~d}$ & $38.7 \mathrm{c}$ & $48 \mathrm{cde}$ & $5.2 \mathrm{bc}$ & $9.9 \mathrm{~d}$ & $69.4 \mathrm{de}$ \\
H-53AE & $10 \mathrm{~d}$ & $78.7 \mathrm{c}$ & $38 \mathrm{e}$ & $44 \mathrm{de}$ & $5 \mathrm{~d}$ & $10.2 \mathrm{a}$ & $69.8 \mathrm{abc}$ \\
H-49AE & $10.5 \mathrm{~d}$ & $79.1 \mathrm{~b}$ & $36.1 \mathrm{~h}$ & $43 \mathrm{e}$ & $4.9 \mathrm{e}$ & $10.1 \mathrm{c}$ & $69.9 \mathrm{ab}$ \\
Tsiri Puma & $11.8 \mathrm{ab}$ & $79.3 \mathrm{a}$ & $38.6 \mathrm{~d}$ & $37 \mathrm{f}$ & $5.3 \mathrm{ab}$ & $10.2 \mathrm{a}$ & $69.1 \mathrm{e}$ \\
Albatros & $11.4 \mathrm{bc}$ & $79.3 \mathrm{ab}$ & $37.3 \mathrm{~g}$ & $35 \mathrm{f}$ & $5.1 \mathrm{~d}$ & $9.8 \mathrm{~d}$ & $69.9 \mathrm{ab}$ \\
Media & 11.2 & 78.2 & 37.7 & 48.3 & 5.1 & 10 & 69.6 \\
DSH & 0.53 & 0.18 & 0.12 & 5.28 & 0.13 & 0.13 & 0.36 \\
\hline
\end{tabular}

Medias con la misma letra en la columna son estadísticamente iguales (Tukey, 0.05). DSH, diferencia significativa honesta. $\mathrm{RG}=$ rendimiento de grano; $\mathrm{PH}=$ peso hectolítrico; $\mathrm{PCG}=$ peso de 100 granos; $\mathrm{IF}=$ índice de flotación; ${ }^{\S}=$ informados a base seca.

La significancia de IGA indica que los híbridos evaluados presentaron diferentes respuestas o adaptación en algún ambiente específico. Para rendimiento, los dos primeros componentes de SREG explicaron $82.7 \%$ (CP1 59.5\% y CP2 23.2\%) de la variabilidad debido a la interacción (Figura 1A). Crossa (1990) menciona que el valor superior a 75\% de CP es aceptable para ser considerada una interpretación confiable de híbrido-ambiente. Siendo resultados consistentes con hallazgos de otros investigadores (Vázquez et al., 2012; Lozano et al., 2015). La longitud larga del vector ambiente implica una mayor diferencia en rendimiento entre los HIB (Crossa et al., 2013).

Los ambientes más alejados fueron TEM y ATL, mientras que los vectores más cercanos al origen correspondieron a Jocotitlán, Jilotepec e Ixtlahuaca. En la localidad de TEM se registró la mayor variabilidad entre HIB ( $>$ log del vector) y mayor rendimiento. En el macro ambiente conformado por TEM, JIL e IXT, destacó el híbrido Atziri Puma al registrar mayor rendimiento, seguidos por los híbridos H-66 y H-50 (Figura 1A) con mejores rendimientos. 

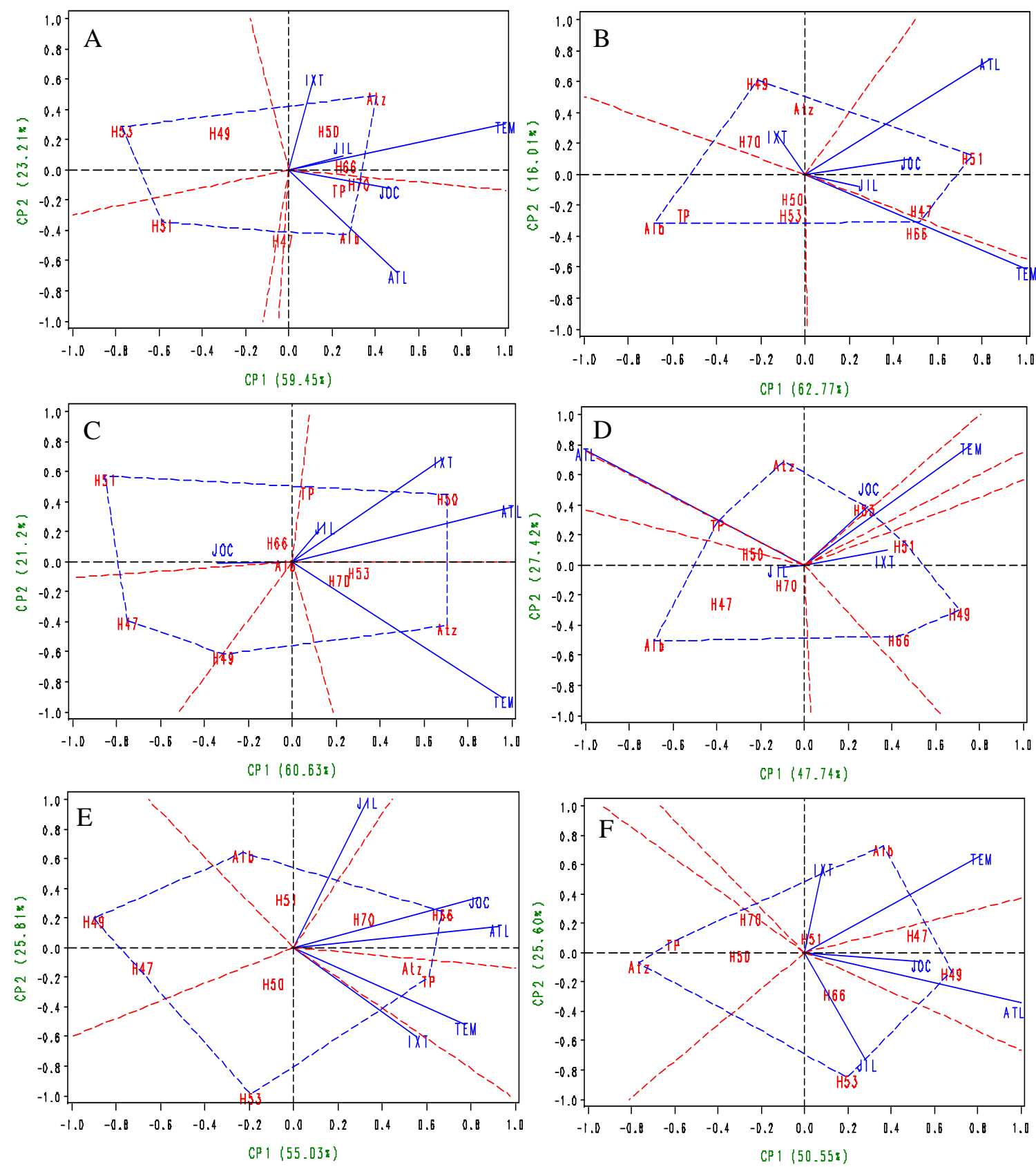

Figura 1. Gráficas biplot de SREG para las variables de rendimiento de grano (A) índice de flotación (B); peso de cien granos (C); porcentaje de almidón (D); aceite (E); y proteína (F).

Los rendimientos oscilaron de 9 a $12.4 \mathrm{t} \mathrm{ha}^{-1}$ (Cuadro 1). Los híbridos H-70, H-66 y Tsiri Puma se adaptaron mejor debido a que presenta el valor del CP1 más cercanos al origen cero (Vázquez et al., 2016), por lo tanto, presentan mayor estabilidad en todos los ambientes de prueba; en paralelo, los más alejados mostraron mayor variación en su comportamiento (Crossa et al., 2015). Según Crossa et al. (2013), desde el punto de vista de un fitomejorador, los mejores híbridos son aquellos con valores de PC2 cercanos a cero y valores altos de PC1. En este caso, los híbridos H-66, H-70 y Tsiri Puma presentan estos atributos, con adaptabilidad y estabilidad de rendimiento para recomendación en otras áreas de Valles Altos del Estado de México. 
En el segundo macro ambiente (ATL y JOC), el híbrido Albatros obtuvo los mejores rendimientos, le siguieron Tsiri Puma y H-70 con rendimientos promedios de 11.8 y $11.6 \mathrm{t} \mathrm{ha}^{-1}$ respectivamente. A pesar de la respuesta positiva de Albatros en este macro ambiente, específicamente en ATL, se observaron rendimientos bajos $\left(9 \mathrm{t} \mathrm{ha}^{-1}\right)$, respecto a los demás ambientes. Respuesta que se atribuye a la baja inversión en fertilización y control fitosanitario (Martínez et al., 2018), lo cual afectó el vigor de las plantas y en el rendimiento de grano.

\section{Características físicas}

El IF se explicó en $78.8 \%$ por los dos primeros CP. La Figura $2 \mathrm{~B}$ muestra en orden ascendente, de izquierda a derecha, el número de granos flotantes; es decir, inverso a la dureza. Según la clasificación de dureza por el IF (SE, 2002), la dureza de los híbridos fue de intermedia a dura (IF). La industria de la masa y la tortilla (IMT) prefiere maíces con IF $\leq 40$ y la de harina nixtamalizada (IHN) con < 20 granos flotantes (Gaytán et al., 2013). La preferencia crece hacia la izquierda del biplot, con IF más bajos (Figura 1B). Los híbridos evaluados presentaron IF de 15-50, que son aptos para la IMT, absorben más agua durante la nixtamalización y reposo, lo anterior, se relacionado con un mayor rendimiento de masa y tortilla (Vázquez et al., 2015).

Dentro del grupo de los granos duros se encuentran el Tsiri Puma y Albatros (Cuadro 1), el resto corresponde a granos de dureza intermedia. Los híbridos de grano duro (Tsiri Puma y Albatros) se asocia con mayores rendimientos, lo cual coincide con lo informado por Vázquez et al. (2012), que entre más duro el grano, mayor será su RG, PH y menor IF. Estos híbridos pueden recomendarse para la industria de harinas nixtamalizadas (IHN), al alcanzar una menor hidratación durante la cocción, facilitando su molienda (Salinas et al., 2012).

El híbrido H-51 AE corresponde al de mayor estabilidad en el IF (Figura 1B) considerando los criterios mencionados por Crossa et al. (2013) en los PC y en Jocotitlán presentó los granos más suaves. Los híbridos Albatros y Tsiri Puma respondieron positivamente a la adaptabilidad en ATL y JOC, mientras que el híbrido H-49 AE en TEM.

En el biplot de IF se observa que en TEM y ATL hubo mayor variabilidad entre los HIB, por lo que los resultados obtenidos en los dos ambientes son más confiables en relación a los otros ambientes de producción, aunque presentan mayor efecto en la IGA que en JOC, donde aparecen cerca del eje X. Yan et al. (2007) explican que, a mayor dispersión relativa de los AMB, con respecto al eje horizontal, se reduce la representatividad de este en el análisis, puesto que hay mayor efecto de IGA en la variable. JIL e IXT no discriminaron entre híbridos, dada su cercanía al origen (vector de menor longitud). Yan et al. (2007) señalan que los ambientes que presentan un ángulo menor de $90^{\circ}$ clasifican a los híbridos de manera semejante, caso similar se muestra en estos dos ambientes (Figura 1B).

El PH tuvo comportamiento semejante a IF, registrando la mejor respuesta en los híbridos Atziri Puma y Albatros (79.3 kg hL $\mathrm{k}^{-1}$ ), seguidos por el híbrido H-49 AE (79.1 kg hL $\left.{ }^{-1}\right)$. Los híbridos que presentaron menor PH fueron H-47 AE, Atziri Puma y H-66 (Cuadro 1). En todos los híbridos evaluados se encontró valor superior al requerido por la NMX-032/1 (SE, 2002) de $74 \mathrm{~kg} \mathrm{hL}^{-1}$. Estos resultados superan a los informados por Vázquez et al. (2016) al evaluar la estabilidad del rendimiento y las variables físicas del grano, así como para nixtamal y tortilla de once híbridos de maíz en seis ambientes en la región central de Valles Altos de México. 
En el tamaño de grano, representado por el peso de cien granos (PCG) es una variable común en la elección de granos a procesar. Las dos primeras PC fueron significativas y representaron $81.83 \%$ de la variabilidad de los datos (Figura 1C). La norma (NMX-034/1 2002) no consideró el tamaño del grano; sin embargo, es una variable de interés que impacta en la cocción y absorción de agua durante la nixtamalización (Antuna et al., 2008).

Considerando la clasificación de Salinas y Vázquez (2006), los granos grandes presentan PCG> 38 $\mathrm{g}$, los intermedios un PCG de 33 a $38 \mathrm{~g}$ y los granos pequeños $\mathrm{PCG}<33 \mathrm{~g}$. Los híbridos Atziri Puma en TEM y H-50 en ATL presentaron los granos más grandes (Figura 1C). No obstante, de acuerdo con los criterios de selección de Salinas y Aguilar (2010), las industrias de masa-tortilla y de harina nixtamalizada prefieren granos pequeños y medianos. Al extender los vectores ambientales hacia su lado negativo, se encontró una alta correlación entre H-51AE en TEM, así como entre H-47AE en IXT y ATL, que muestra una adaptación específica a dichos ambientes, con granos de tamaño pequeño (Figura 1C).

La IHN antepone maíces de tamaño intermedio, en tanto que IMT prefiere granos intermedios y pequeños, por la mejor hidratación que favorece el rendimiento de nixtamal, masa y tortilla al presentar humedad elevada (Salinas y Aguilar, 2010). Bajo esta premisa siete de los diez híbridos evaluados presentaron tamaño intermedio, resultando una alternativa con adecuadas características para su procesamiento para las IMT.

\section{Características químicas}

Existen diferencias significativas ( $p<0.05$ ), para HIB, AMB y se detectó interacción entre los dos factores en las variables evaluadas, se observó una correlación entre PROT $v s$ ACEI $(\mathrm{r}=0.6, p \leq$ $0.01)$, ACEI $v s$ ALM ( $\mathrm{r}=-0.86, p \leq 0.01)$ y PROT $v s$ ALM ( $\mathrm{r}=-0.82, p \leq 0.01)$, que confirma lo reportado por Vázquez et al. (2015) y es visible en los biplot, pues son similares o 'cuasisimétricas' unas con otras.

El compuesto más importante del grano de maíz es el almidón, ya que representa 3/4 partes del total (Paredes et al., 2009). En este estudio se encontraron porcentajes de ALM (69-70\%) con una mínima diferencia entre los híbridos. Estos valores son consistentes con lo reportado para maíces tipo dentado (Watson, 2003). El hibrido H-49 AE resultó mejor adaptado, en relación con los otros híbridos y con porcentaje mayor de almidón, seguidos por H-47 AE, H-53 AE y Albatros, mientras que el Atziri Puma y Tsiri Puma presentaron valores $<69.1$, respectivamente (Cuadro 1).

Los híbridos destacan en su contenido de ACE, los menores porcentajes fueron de 4.88 y $4.9 \%$ (Cuadro 1), mayor a lo observado en híbridos de endospermo normal por Vázquez et al. (2015). Los mayores porcentajes de ACE se tuvieron en granos más grandes $(r=0.43, p \leq 0.01)$. De acuerdo con Vázquez et al. (2015), son considerados híbridos de alto contenido de aceite (ACA) cuando poseen más de 5\%, en el grano. Dentro de esta clasificación se puede ubicar los HIB probados en este experimento, lo que es relevante en la elaboración de tortillas por la IMT, por la relación entre el ACE en grano y la suavidad de las tortillas resultantes (Vázquez et al., 2016), mayor cantidad de éste disminuye la retrogradación de los almidones (Putseys et al., 2010). 
Por lo tanto, la presencia de aceite en el grano de maiz puede mejorar significativamente la firmeza y la masticación de la tortilla (Vidal-Quintanar et al., 2001). En JOC y ATL se produjeron granos con mayor porcentaje de aceite. Los híbridos con porcentaje altos de aceite en grano fue H-66, Atziri Puma, y Tsiri Puma, estos dos últimos se muestran estables en TEM. Los híbridos con menor porcentaje fue H-49 AE y H-47 AE con 4.9\% respectivamente (Figura 1E).

En proteína se obtuvo entre 9.75 y $10.31 \%$ (Cuadro 1), los porcentajes mayores en H-53 AE, Tsiri Puma y Atziri Puma ( $\geq 10.22 \%$ ), mientras que el menor se obtuvo en el híbrido Albatros. Los resultados son semejantes a lo informado por Vázquez et al. (2012) que observaron de 9.76 a $12.54 \%$ de proteína en variedades locales y en híbridos mejorados de 8.24 a $11.34 \%$. Estas variaciones en las proteínas están asociadas con la genética de los materiales evaluados.

\section{Calidad nixtamal-tortillas}

Se presentaron diferencias significativas $(p<0.05)$ entre los híbridos, ambientes y en IGA para las variables de calidad de nixtamal, masa y tortillas. La asignación de tiempos de cocción de acuerdo con el IF permitió alcanzar humedades en el nixtamal entre $45.6 \%$ (intermedios) y $47.3 \%$ (duros), valores que en masa se incrementaron de 57.4 y $58.1 \%$ (Cuadro 2), aumento que se debe, en los maíces duros, al mayor tiempo de cocción, y en la masa a la adición de agua durante la molienda y para acondicionarla, previo al troquelado.

Cuadro 2. Valores promedio de variables de calidad de nixtamal, masa y tortillas de 10 híbridos de maíz probados en cinco ambientes de Valles Altos del Estado de México. Primaveraverano 2016.

\begin{tabular}{|c|c|c|c|c|c|c|c|c|c|}
\hline \multirow{3}{*}{ Híbridos } & \multicolumn{5}{|c|}{ Humedad (\%) } & \multirow{3}{*}{$\begin{array}{c}\text { Pérdida } \\
\text { de materia } \\
\text { seca }(\%)\end{array}$} & \multicolumn{3}{|c|}{ Rendimiento ( $\mathrm{kg} \mathrm{kg}^{-1}$ grano) } \\
\hline & \multirow{2}{*}{ Nixtamal } & \multirow{2}{*}{ Masa } & \multicolumn{3}{|c|}{ Tortillas } & & \multirow{2}{*}{ Nixtamal } & \multirow{2}{*}{ Masa } & \multirow{2}{*}{ Tortilla fría } \\
\hline & & & Actual & $24 \mathrm{~h}$ & $48 \mathrm{~h}$ & & & & \\
\hline $\mathrm{H}-51 \mathrm{AE}$ & $46.9 \mathrm{~b}$ & $58.1 \mathrm{a}$ & $44.2 \mathrm{ab}$ & $45.3 \mathrm{abc}$ & 44.8 cde & $3.35 \mathrm{ef}$ & $1.89 \mathrm{c}$ & $2.01 \mathrm{a}$ & $1.55 \mathrm{a}$ \\
\hline $\mathrm{H}-47 \mathrm{AE}$ & $46.1 \mathrm{~d}$ & $57.8 \mathrm{ab}$ & $44.7 \mathrm{a}$ & 45 bcde & 44.8 bcde & $3.46 \mathrm{~cd}$ & $1.88 \mathrm{~d}$ & $2 \mathrm{abc}$ & $1.57 \mathrm{a}$ \\
\hline H-66 & $46.5 \mathrm{c}$ & $57.7 \mathrm{abc}$ & $45.1 \mathrm{a}$ & $45.3 \mathrm{abcd}$ & $45.4 \mathrm{ab}$ & $3.54 \mathrm{c}$ & $1.9 \mathrm{a}$ & 1.99 bcde & $1.56 \mathrm{a}$ \\
\hline ATZ & $46.1 \mathrm{~d}$ & $57.5 \mathrm{bc}$ & $44.9 \mathrm{a}$ & 44.7 de & $44.5 \mathrm{def}$ & $3.28 \mathrm{f}$ & $1.87 \mathrm{f}$ & 1.99 bcde & $1.57 \mathrm{a}$ \\
\hline $\mathrm{H}-50$ & 45.7 ef & $57.5 \mathrm{bc}$ & $44.7 \mathrm{a}$ & 44.9 bcde & 44.5 def & 3.34 ef & $1.85 \mathrm{j}$ & $1.99 \mathrm{cde}$ & $1.52 \mathrm{a}$ \\
\hline $\mathrm{H}-70$ & $45.6 \mathrm{f}$ & $57.4 \mathrm{c}$ & $43.5 \mathrm{~b}$ & $44.6 \mathrm{e}$ & $44.1 \mathrm{f}$ & $3.38 \mathrm{de}$ & $1.87 \mathrm{~g}$ & $1.98 \mathrm{e}$ & $1.52 \mathrm{a}$ \\
\hline H-53AE & $45.6 \mathrm{f}$ & $57.6 \mathrm{bc}$ & $44.6 \mathrm{a}$ & 44.8 cde & $44.9 \mathrm{bcd}$ & $3.48 \mathrm{c}$ & $1.85 \mathrm{i}$ & $1.98 \mathrm{de}$ & $1.55 \mathrm{a}$ \\
\hline H-49AE & $46 \mathrm{de}$ & $58 \mathrm{a}$ & $44.7 \mathrm{a}$ & $45.7 \mathrm{a}$ & $45.5 \mathrm{a}$ & $3.94 \mathrm{a}$ & $1.87 \mathrm{~h}$ & 1.99 bcde & $1.54 \mathrm{a}$ \\
\hline $\mathrm{TP}$ & $46.4 \mathrm{c}$ & $58.1 \mathrm{a}$ & $44.9 \mathrm{a}$ & $45.4 \mathrm{ab}$ & $45.1 \mathrm{abc}$ & $3.73 \mathrm{~b}$ & $1.89 \mathrm{~b}$ & 2 abcd & $1.55 \mathrm{a}$ \\
\hline ALB & $47.3 \mathrm{a}$ & $57.9 \mathrm{a}$ & $44.4 \mathrm{ab}$ & 45.2 bcde & $44.3 \mathrm{ef}$ & $3.53 \mathrm{c}$ & $1.88 \mathrm{e}$ & $2.01 \mathrm{ab}$ & $1.53 \mathrm{a}$ \\
\hline DSH & 0.28 & 0.35 & 1.08 & 0.57 & 0.56 & 0.08 & 0.01 & 0.02 & 0.07 \\
\hline
\end{tabular}

Medias con la misma letra minúscula en la columna son estadísticamente iguales (Tukey, 0.05). DSH= diferencia significativa honesta. 
El mayor rendimiento en masa se encontró en H-51 AE, seguido de H-47 AE, Albatros y Tsiri Puma con $2 \mathrm{~kg}$ de masa por cada $\mathrm{kg}$ de maíz nixtamalizado. El rendimiento de nixtamal y masa fue semejante a lo informado por Gaytán et al. (2013); Vázquez et al. (2015); Vázquez et al. (2016). Mientras que la humedad en tortillas recién elaboradas (30 min) fue de 43.5 a $45.1 \%$ (Cuadro 2). La localidad de JOC mostró la mayor diversidad en la HT 30 min entre híbridos, los híbridos H-51 AE y Atziri Puma fueron los más estables en la HT 30 min.

La HT almacenadas ( 24 y 48 h) fue ligeramente superior a las recién elaboradas, en los híbridos (Cuadro 2). En TEM los híbridos Tsiri Puma, Albatros y H-53 AE presentaron altos porcentajes de humedad, y el más bajo en el híbrido H-50 (Cuadro 2). En todos los híbridos el rendimiento de tortillas, fue superior al $1.5 \mathrm{~kg}$ de tortilla por $\mathrm{kg}$ de grano de maíz demandado por los industriales de la masa-tortillas (Salinas y Aguilar 2010). Por ambiente, el rendimiento promedio más bajo se observó en Jilotepec con $1.51 \mathrm{~kg}$ de tortilla por kg de grano de maíz ${ }^{-1}$. Mientras que el hibrido H70 presentó valores menores y el más bajo en JOC, aunque se encuentra mayor adaptado a JIL.

En la pérdida de materia seca (PMS), todos los materiales registraron valores inferiores a los $5 \mathrm{~g}$ $100 \mathrm{~g}^{-1}$ declarado en la norma -034(1) (SE, 2002) (Cuadro 2). Los resultados coinciden con lo

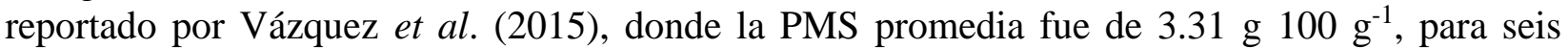
híbridos) de Valles Altos de México.

Los consumidores de tortillas demandan que estas sean suave y flexibles (Mao et al., 2002). La mayoría de las variables de textura mostraron diferencia significativa $(p>0.01)$ por efecto del genotipo, del ambiente y en la IGA, los resultados expuestos incluyen los promedios de las cinco localidades. Las tortillas recién elaboradas mostraron mayor variabilidad, respecto a las almacenadas (Figura 1).

A los 30 min de elaboradas las tortillas más suaves, fueron las de los híbridos H-47E, H-53AE y $\mathrm{H}-49 \mathrm{AE}$, que requirieron menos fuerza para romperse $(\mathrm{x}=1.4 \mathrm{~N})$, tuvieron una buena elongación $(\mathrm{EL})(\mathrm{x}=8.3 \mathrm{~mm})$ y su módulo de elasticidad $(\mathrm{Y})$ fue el más bajo $\left(\mathrm{x}=11.8 \mathrm{~N} \mathrm{~cm}^{-2}\right)$, esta calidad de las tortillas también puede relacionarse con su contenido de aceite $( \pm 5 \%)$, según lo informaron Vázquez et al. (2015). El siguiente grupo incluyó al H-51AE, H-66, H-50 y Tsiri-Puma, cuyas tortillas requirieron $1.56 \mathrm{~N}$ para romperse, el $\mathrm{Y}$ se incrementó $\left(\mathrm{x}=12.4 \mathrm{~N} \mathrm{~cm}^{-2}\right)$ de manera proporcional al aumento de la FZA, ya que su EL fue igual a la del grupo 1 (8.3 mm) (Figura 3).

(A)

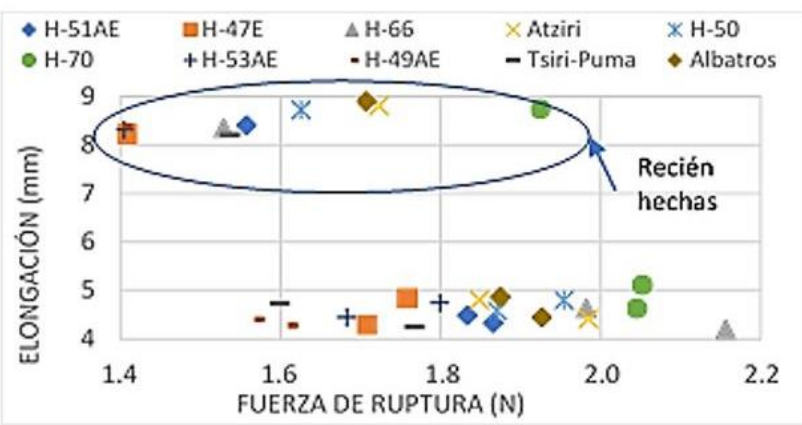

(B)

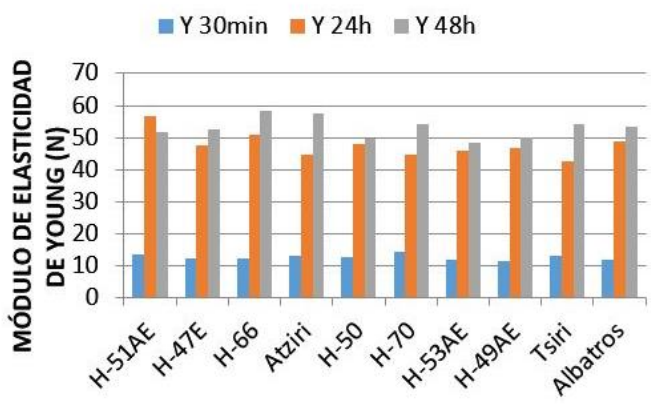

Figura 3. Fuerza de ruptura y elongación (A) y módulo de elasticidad de Young (B) en tortillas recién elaboradas y almacenadas ( 24 y 48 h). Promedios de las cinco localidades. 
La pérdida de flexibilidad de las tortillas al enfriarse ( 24 y 48 h en refrigeración) se debe a la formación de una estructura rígida causada por la retrogradación del almidón y la asociación con proteínas, fibra y otros componentes químicos (Agama-Acevedo et al., 2011). El mayor aumento de la FZA ocurrió a las $24 \mathrm{~h}$, este fue del orden de $0.28 \mathrm{~N}(18 \%)$, en tanto a las $48 \mathrm{~h}$ de almacenamiento en algunas tortillas se redujo la FZA, en otras se mantuvo igual y solamente en tres genotipos H-66, Atsiri-Puma y Tziri-Puma se incrementó la FZA. Comportamiento semejante se observó en el Y. La elongación de las tortillas almacenadas no mostro diferencia estadística por efecto del almacenamiento, los valores fueron de 4.3-5.1 $\mathrm{mm}(24 \mathrm{~h}) \mathrm{y}$ de $4.2-4.6 \mathrm{~mm}(48 \mathrm{~h})$.

Las mejores tortillas con $24 \mathrm{~h}$ de almacenamiento, fueron del híbrido $\mathrm{H}-49 \mathrm{AE}$, estas requirieron menos fuerza para romperse $\left(x=1.6 \mathrm{~N}\right.$ ), su EL fue de $4.4 \mathrm{~mm}$ y su $\mathrm{Y}$ de $46.8 \mathrm{~N} / \mathrm{cm}^{2}$ (Figura $3 \mathrm{~A}$ y B). La buena calidad de las tortillas almacenadas podría explicarse por el contenido de aceite de estos maíces $( \pm 5 \%)$. Al respecto se ha informado (Vázquez et al., 2015) que durante la nixtamalización los lípidos interactúan con las moléculas de amilosa, afectando las propiedades fisicoquímicas del almidón y favoreciendo la formación del complejo amilos-lípido, el cual favorece la textura de las tortillas (Vázquez et al., 2014).

\section{Conclusiones}

El mayor rendimiento y variabilidad entre híbridos se registró en la localidad de Temascalcingo. En el macro ambiente formado por las localidades de TEM, JIL e IXT, el mayor rendimiento fue para los híbridos Atziri Puma, H-66 y H-50, fueron los de mayor adaptabilidad y estabilidad, por lo que se pueden recomendar ampliamente para Valles Altos del Estado de México. En el segundo macro ambiente (ATL y JOC) destacaron Tsiri Puma y H-70 con rendimientos promedios de 11.8 y $11.6 \mathrm{t} \mathrm{ha}^{-1}$ respectivamente. Todos los híbridos cumplieron con las especificaciones comerciales de la norma NMX-034(1) para maíces destinados al proceso de nixtamalización.

Son granos de tamaño, mediano a pequeño, con peso hectolitrico por arriba de $74 \mathrm{~kg} \mathrm{hL}^{-1}$ y dureza de intermedia a dura. Su contenido de aceite estuvo entre 4.9 y $5.4 \%$. Las pérdidas de materia seca en el nejayote, estuvieron dentro de lo demandado por los industriales, en tanto que las humedades en nixtamal, masa y tortillas, así como los rendimientos fueron elevadas. Las mejores tortillas recién elaboradas se produjeron con los híbridos androesteriles H-47AE, H-53AE, H-49AE que fueron los de menor rendimiento $\left(\mathrm{x}=10.5 \mathrm{t} \mathrm{ha}^{-1}\right)$. Los resultados de esta investigación permiten recomendar estos nuevos híbridos, por su estabilidad en el rendimiento, en los ambientes de prueba, calidad comercial del grano y calidad de sus tortillas.

\section{Literatura citada}

AACCI-American Association of Cereal Chemists International. 2000. Method 44-15.02. Moisture 46-11.02. Determination of Crude Protein; 76-13.01 Total stach (AACCI, Ed) St. Paul, MN, E.E.U.U.: AACC International.

Agama-Acevedo, E.; Salinas-Moreno, Y.; Pacheco-Vargas, G. y Bello-Pérez, L. A. 2011. Características físicas y químicas de dos razas de maíz azul: morfología del almidón. Rev. Mex. Ciencias Agric. 2(3):317-329. 
Antuna, G. O.; Rodríguez, H. S.; Arámbula, V. G.; Palomo, G. A.; Gutiérrez, A. E.; Espinosa, B. A.; Navarro, O. E. y Andrio, E. E. 2008. Calidad nixtamalera y tortillera en maíces criollos de México. Rev. Fitotec. Mex. 31(3):23-27.

Crossa, J. 1990. Statistical analysis of multilocation trials. Adv. Agron. 44(1):55-85. doi:10.1016/S0065-2113(08)60818-4.

Crossa, J.; Vargas, M.; Cossani, C. M.; Alvarado, J.; Burgueño, G.; Mathews, K. L. and Reynolds, M. P. 2013. Evaluation and interpretation of interactions. Agron. J. 107(1):736-737. doi:10.2134/agronj2012.049.

Crossa, J.; Vargas, M.; Cossani, M.; Alvarado, G.; Burgueño, J.; Mathews, K. L. and Reynolds, M. 2015. Evaluation and interpretation of interactions. Agro. J. 107(2):736-747.

Gaytán, M. M.; Reyes. V. M.; Figueroa, C. J.; Morales, S. E. y Rincón, S. F. 2013. Selección de maíces criollos para su aplicación en la industria con base en su valor agregado. Rev. Fitotec. Mex. 36(3-A):339-346.

Lozano, R. A.; Santacruz, V. A.; San Vicente, G, F.; Crossa, J.; Burgueño, J. y Molina, G. J. D. 2015. Modelación de la interacción híbrido X ambiente en rendimiento de híbridos de maíz blanco en ambientes múltiples. Rev. Fitotec. Mex. 38(4):337-347.

Mao, Y.; Flores, R. A. and Loughin, T. M. 2002. Objective texture measurement of commercial wheat flour tortillas. Cereal Chem. 79(5):648-653.

Martínez, G. A. B.; Zamudio, G. M.; Tadeo, R. A.; Espinosa, C. J.; Cardoso, G. G.; Vázquez, C. y Turrent, F. A. 2018. Rendimiento de híbridos de maíz grano blanco en cinco localidades de Valles Altos de México. Rev. Méx. de Cienc. Agríc. 9(7):1447-1458.

NMX-034/1-SCFI-2002. Maíz para consumo humano. México, DF. Secretaría de Economía.

Paredes, L. F.; Guevara, L. A. y Bello, P. A. 2009. La nixtamalización y el valor nutritivo del maíz. Ciencias. 93:60-70.

Putseys, J. A.; Derde, L. J.; Lamberts, L.; Östman, E.; Björck, I. and Delcour, J. A. 2010. Functionality of short chain amylose-lipid complexes in starch-water system and their impact on in vitro starch degradation. J. Agri. Food Chem. 58(3):1939-1945.

Salinas, M. Y. y Aguilar, M. L. 2010. Efecto de la dureza del grano de maíz (Zea mays L.) sobre el rendimiento y calidad de la tortilla. Ingeniería Agrícola y Biosistemas. 2(1):5-11.

Salinas, M. Y. y Vázquez, C. G. 2006. Metodologías de análisis de calidad nixtamalero-tortillera de maíz. 1a ed. México: Instituto Nacional de Investigaciones Forestales Agrícolas y Pecuarias (INIFAP). Folleto técnico núm. 23.98 p.

Salinas, M. Y.; Vázquez, C. M. G.; Velázquez, C. G. A. and Soria, R. J. 2012. Maize quality selection scheme for masa, tortillas and nixtamalized flours: case of the State of Mexico. Folleto técnico No. 50. INIFAP, Texcoco, Estado de México. 67 p.

SAS Institute. 2002. The SAS system for Windows user's guide. Release 9.0. SAS Institute, Cary, $\mathrm{NC}$.

SIAP-SAGARPA. 2018. Servicio de Información Agroalimentaria y Pesquera-Secretaría de Agricultura, Ganadería, Desarrollo Rural, Pesca y Alimentación. Información básica, www.siap.sagarpa.gob.mx/index.php?option=com_content $\& v i e w=\operatorname{article} \& i d=10 \&$ Itemid $=15$.

Vázquez, C. M. G.; Rojas, M. I.; Santiago, R. D.; Arellano, V. J. L.; Espinosa, C. A.; García, P. M. and Crossa, J. 2016. Stability analysis of yield and grain quality traits for nixtamalization process of maize genotypes cultivated in the Central High Valleys of Mexico. Crop Sci. 56(1):3090-3099. 
Vázquez, C. M. G.; Santiago, R. D.; Gaytán, M. M.; Morales, S. E. and Guerrero, H. M. J. 2015. High oil content maize: Physical, thermal and rheological properties of grain, masa and tortillas. LWT-Food. Sci. Tech. 60(1):156-161.

Vázquez, C. M. G.; Santiago, R. D.; Salinas, M. Y.; Rojas, M. I.; Arellano, V. J. L.; Velázquez, C. G. A. y Espinosa, C. A. 2012. Interacción híbrido-ambiente del rendimiento y calidad de grano y tortilla de híbridos de maíz en Valles Altos de Tlaxcala, México. Rev. Fitotec. Méx. 35(3):229-237.

Vázquez-Carrillo, M. G.; Santiago-Ramos, D.; Salinas-Moreno, Y.; López-Cruz, J.; YbarraMoncada, M. C. y Ortega-Corona, A. 2014. Genotipos de maíz (Zea mays L.) con diferente contenido de aceite y su relación con la calidad y textura de la tortilla. Agrociencia. 48(2):159-172.

Vidal-Quintanar, R. L.; Love, J. and L. A. Johnson. 2001. Role of oil on physical properties of corn masa flours and sensory characteristics of corn tortilla. J. Food Proc. Preserv. 1(25):1-14.

Watson, S. A. 2003. Description, development, structure, and composition of the corn kernel. Chapter 3. In: corn: chemistry and technology. White, P. J. and Johnson, L. A. (Eds.). $2^{\text {nd }}$ edition. American Association of Cereal Chemists, Inc. St. Paul Minnesota, USA. 69-106 pp.

Yan, W.; Kang, M. S.; Ma, B.; Woods, S. and Cornelius, P. L. 2007. GGE biplot vs AMMI analysis of genotype-by-environment data. Crop Sci. 2(47):643-655. 